\title{
Twin-plate Ice Nucleation Assay (TINA) with infrared detection for high- throughput droplet freezing experiments with biological ice nuclei in lab- oratory and field samples
}

Anna T. Kunert et al.

Correspondence to: Janine Fröhlich-Nowoisky (j.frohlich@mpic.de) and Mira L.Pöhlker (m.pohlker@mpic.de)

The copyright of individual parts of the supplement might differ from the CC BY 4.0 License. 
(a)
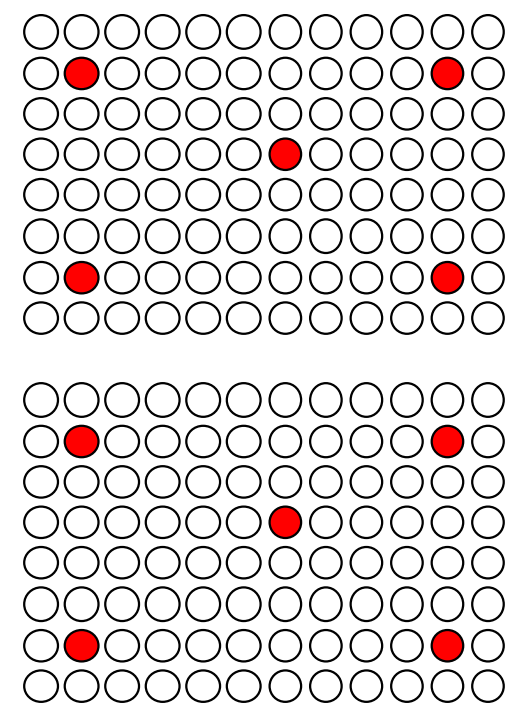

(b)
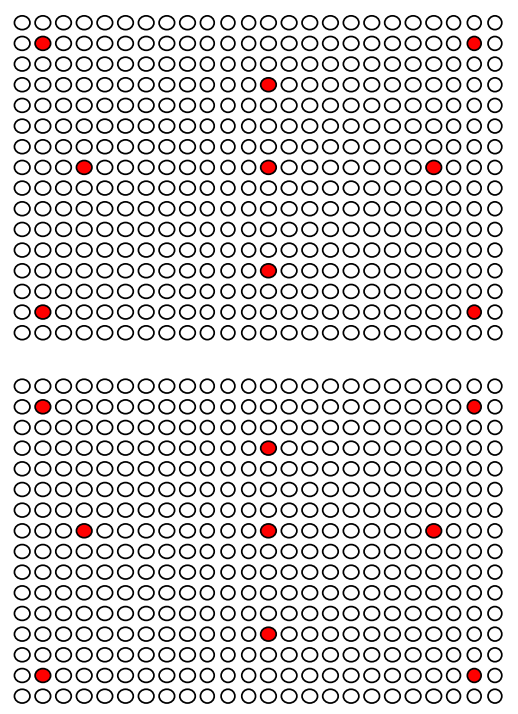

(c)

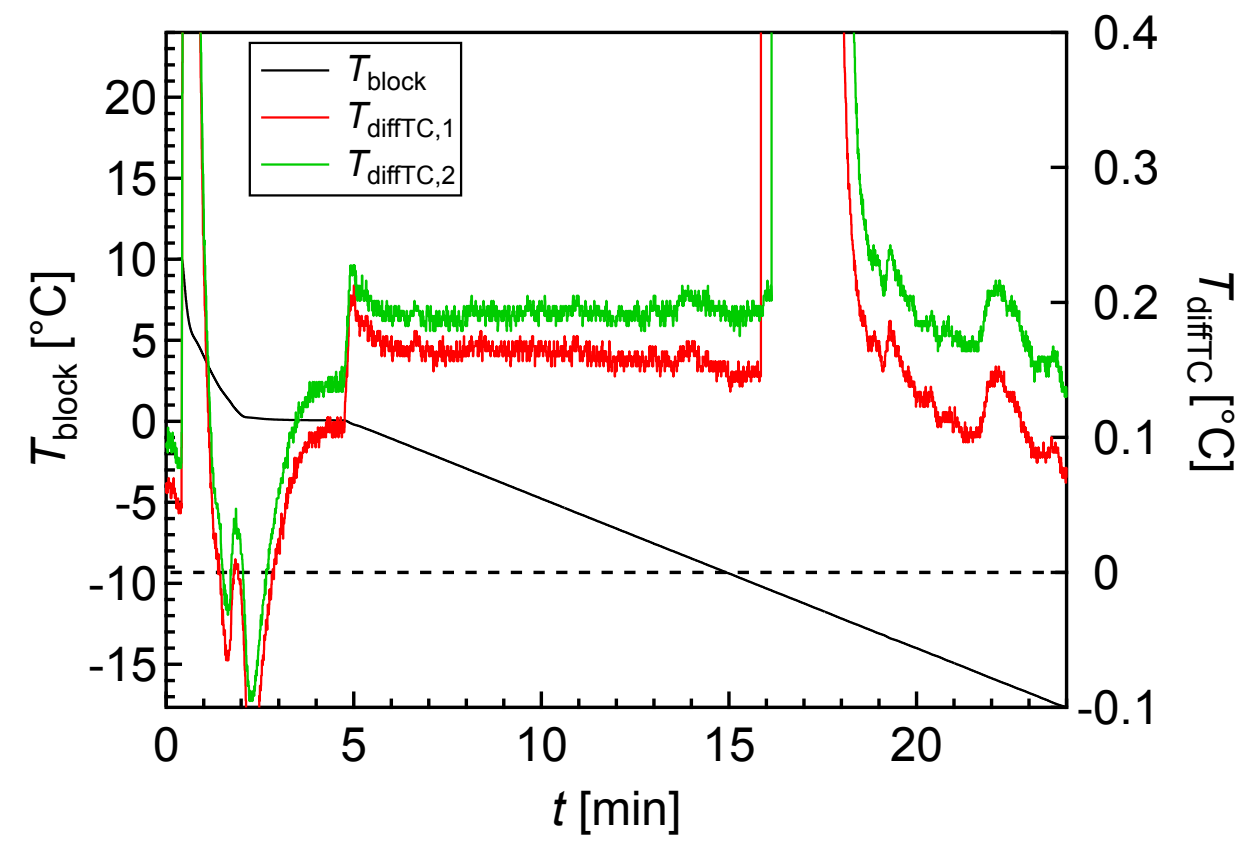

Figure S1. Measurements to determine a potential temperature gradient of the sample holder blocks. Thermocouple measurements were performed in red-marked wells of (a) 96-well block and (b) 384-well block. (c) Measurement of temperature offset between sample holder block (black line) and wells for a continuous cooling rate of $1 \mathrm{~K} \mathrm{~min}^{-1}$ exemplarily for two different wells (green and red lines). 


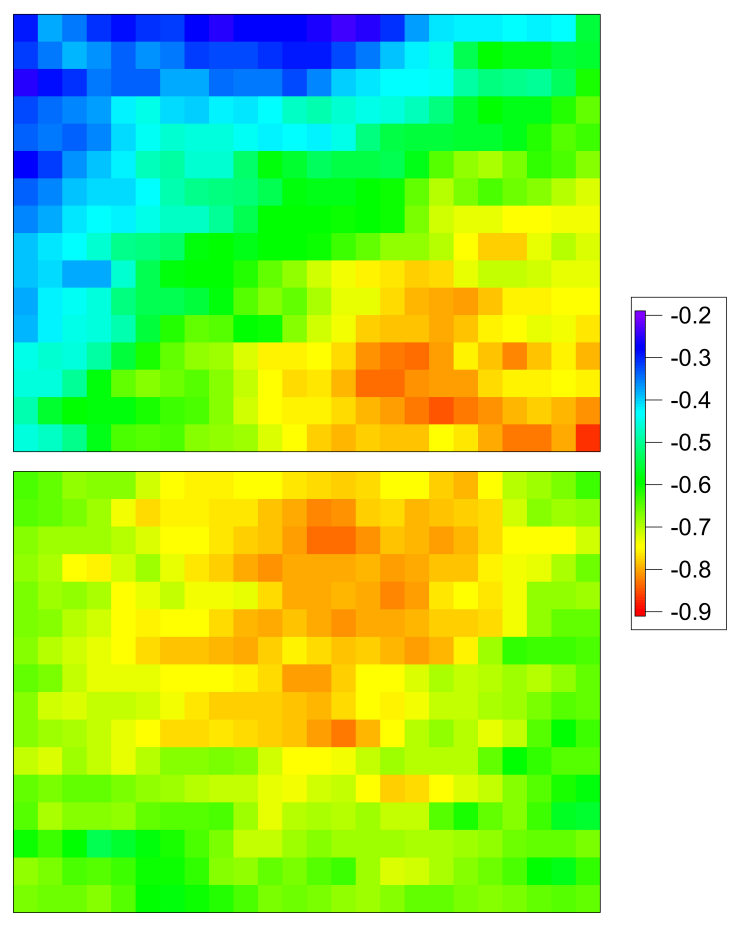

Figure S2. Temperature gradient of the 384-well sample holder block based on thermocouple measurements and 2D interpolation. 


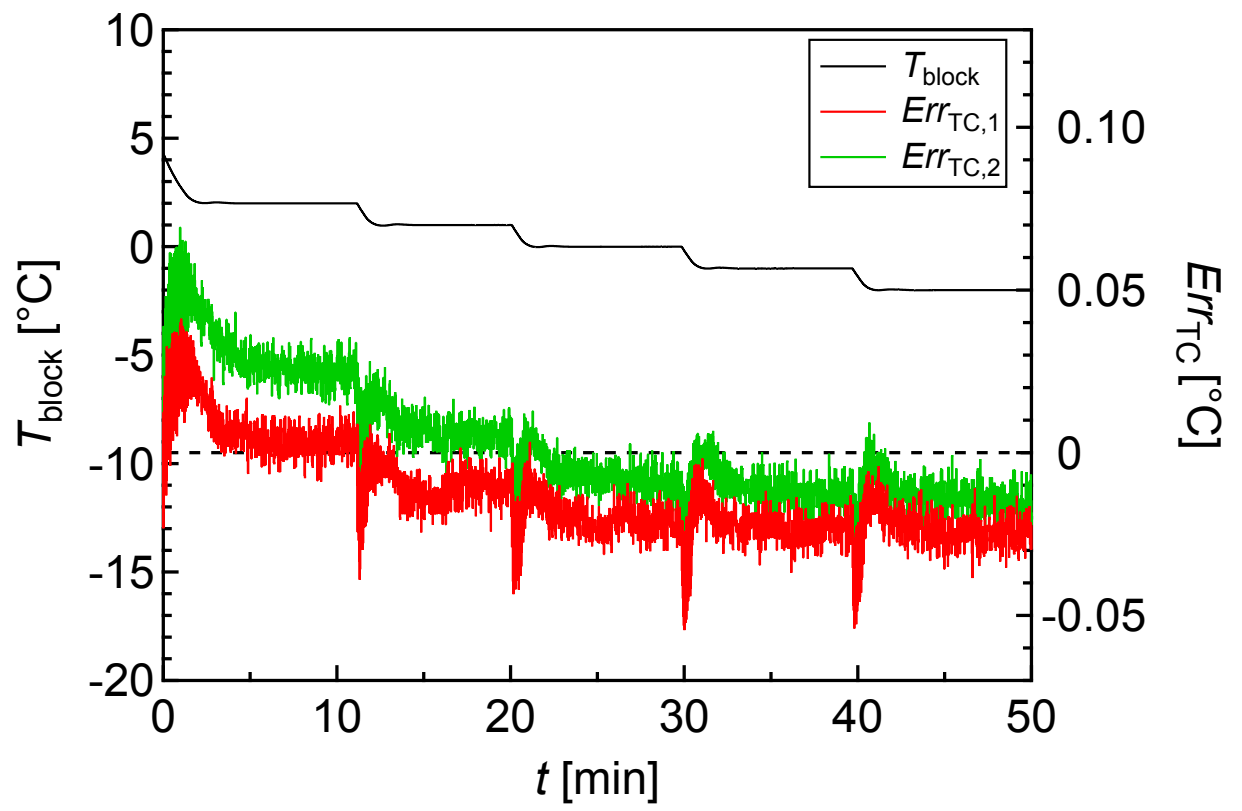

Figure S3. Uncertainty determination of thermocouple measurement. Sample holder block was cooled down (black line), while the difference between ice water and block temperature was monitored for both thermocouples (green and red lines). 

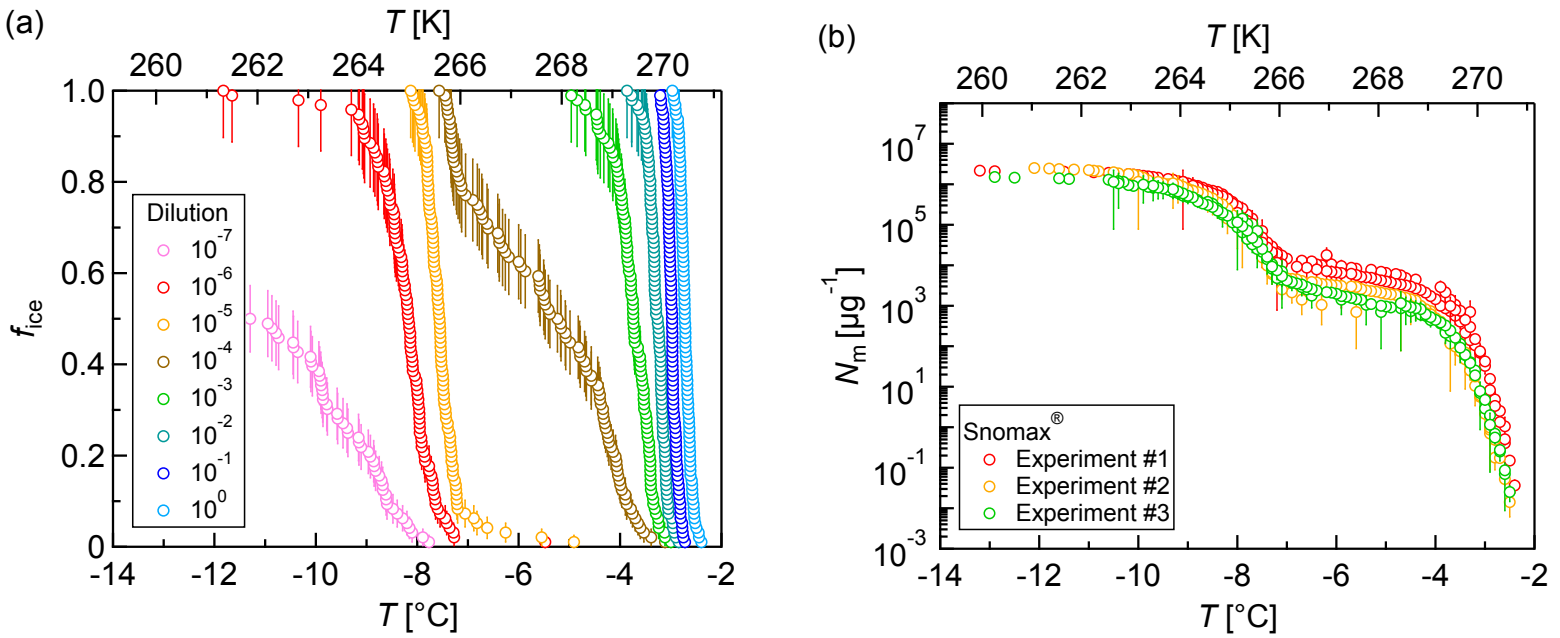

Figure S4. Measurements of dilution series of bacterial IN $\left(\operatorname{Snomax}^{\circledR}\right)$. (a) Fraction of frozen droplets $\left(f_{\text {ice}}\right)$ vs. temperature $(T)$ for each dilution (different symbol color) exemplarily for one experiment. (b) Cumulative number of IN $\left(N_{\mathrm{m}}\right)$ per unit mass of Snomax ${ }^{\circledR}$. Symbol colors indicate three independent measurements. The error bars were calculated using the counting error and the Gaussian error propagation. The temperature error is $0.2 \mathrm{~K}$. 
(a)

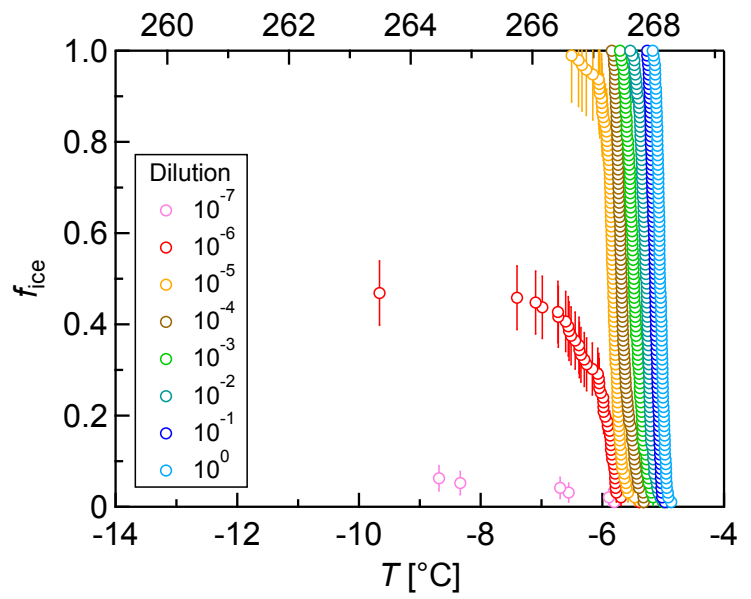

(b)

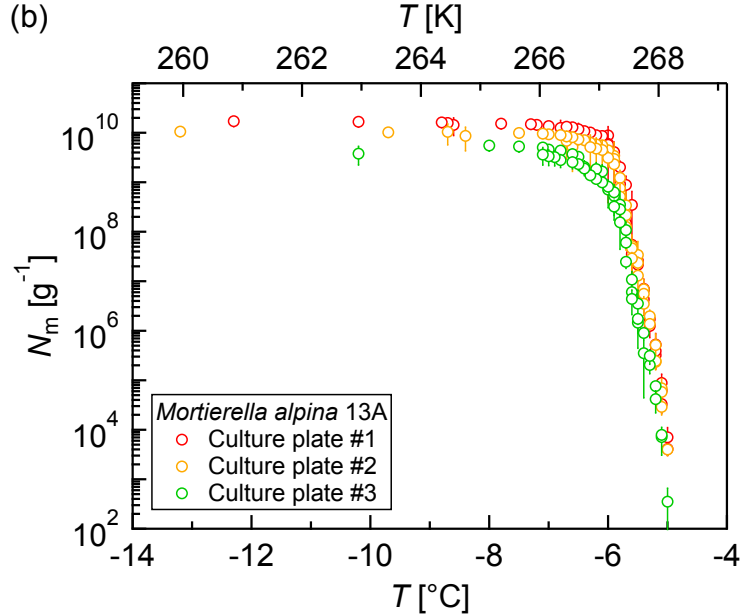

Figure S5. Measurements of dilution series of fungal IN filtrate of Mortierella alpina 13A. (a) Fraction of frozen droplets $\left(f_{\text {ice}}\right)$ vs. temperature $(T)$ for each dilution (different symbol color) exemplarily for one culture plate. (b) Cumulative number of $\mathrm{IN}\left(N_{\mathrm{m}}\right)$ per unit mass of mycelium. Symbol colors indicate three independent measurements of IN filtrate of three different fungal culture plates. The error bars were calculated using the counting error and the Gaussian error propagation. The temperature error is $0.2 \mathrm{~K}$. 
(a)

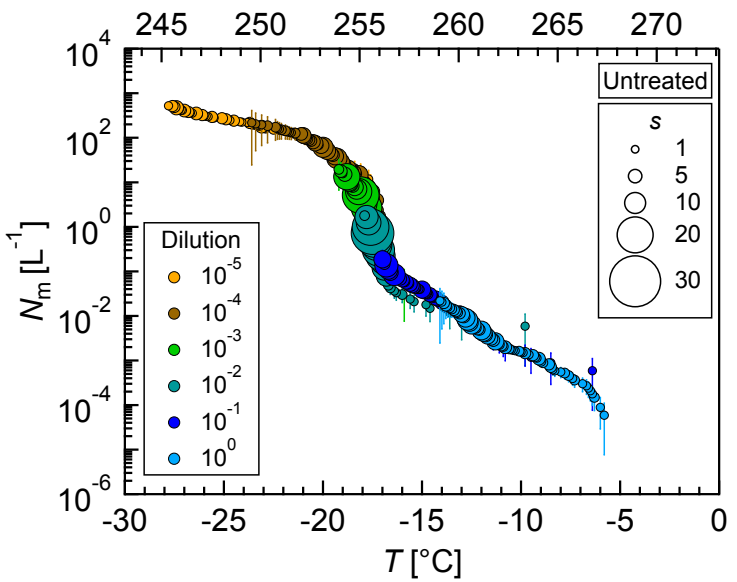

(c)

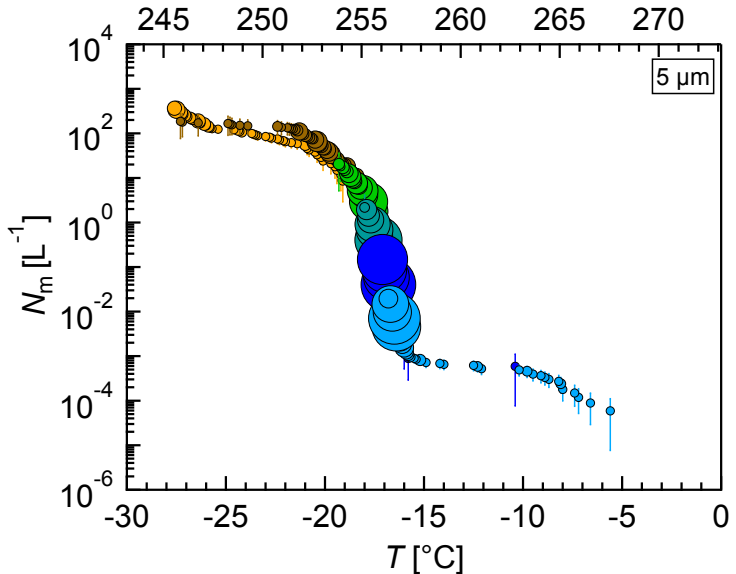

(b)

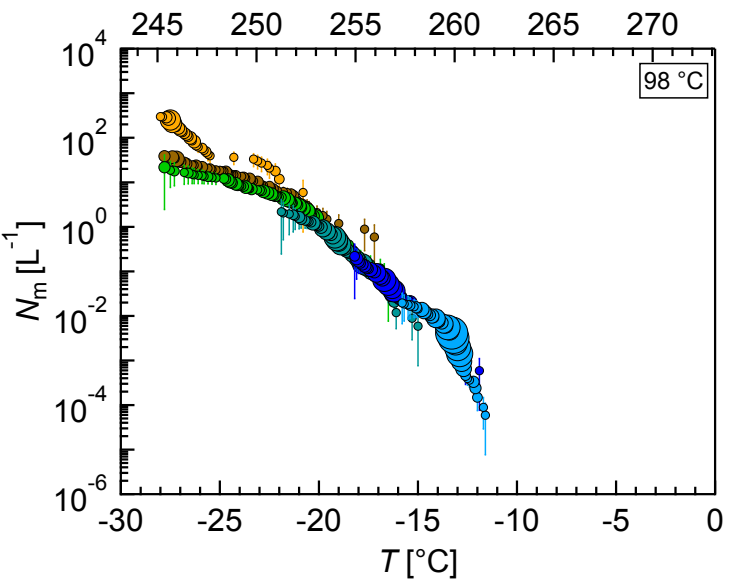

(d)

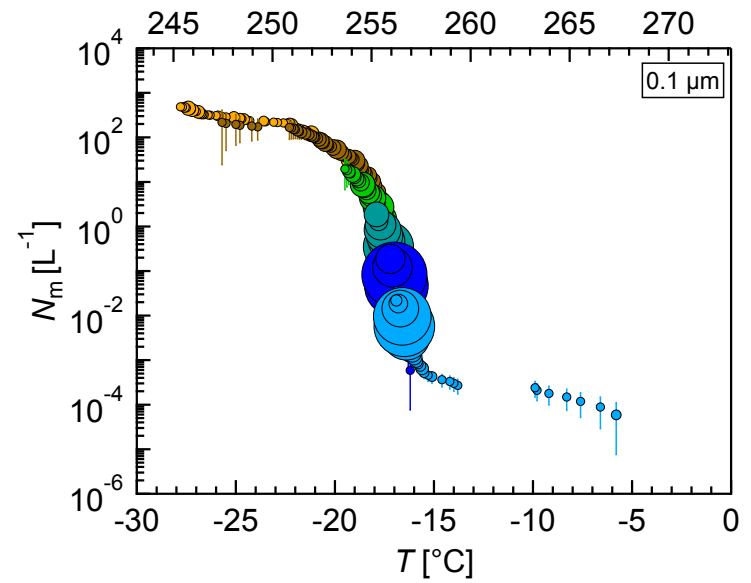

Figure S6. Measurements of dilution series of (a) untreated, (b) heated, (c) $5 \mu \mathrm{m}$ filtered, (d) $0.1 \mu \mathrm{m}$ filtered extracts of atmospheric aerosols. Cumulative number of IN per liter air $\left(N_{\mathrm{m}}\right) \mathrm{vs}$. temperature $(T)$ for different treatments. Droplets of the same dilution of two aliquots were added to a total droplet number of 192 ( 2 x 96 droplets). Symbol colors indicate different dilutions; symbol size indicates the number of frozen droplets per $0.1 \mathrm{~K}$ bin $(s)$. The error bars were calculated using the counting error and the Gaussian error propagation. The temperature error is $0.2 \mathrm{~K}$. 